\title{
Fatores diferenciais e estruturais na localização e crescimento da população rural no oeste do Paraná
}

\author{
Ricardo Rippel \\ Jandir Ferrera de Lima*
}

\begin{abstract}
RESUMO - O objetivo desse artigo é analisar os fatores diferenciais e estruturais que influenciam na localização da população urbana e rural nos municípios do Oeste paranaense no período de 1970 a 2000. Para alcançar esses objetivos utilizou-se o método diferencial estrutural, também conhecido como shift-share, pois o mesmo quantifica o padrão de crescimento e localização da população por domicílio entre os municípios.
\end{abstract}

Palavras-chave: Análise Regional. População. Paraná. Desenvolvimento urbano. Desenvolvimento rural.

\section{INTRODUÇÃO}

Nos anos 1970, o Oeste paranaense passou por uma profunda mudança tecnológica na sua base produtiva agrária, fato que propiciou a ocupação de novas áreas e reestruturação das tradicionais, ocasionando uma forte migração rural para os grandes centros urbanos do Estado e da região e, principalmente, para outros estados (PIFFER, 1999). Nesta década iniciou-se, um processo de rápida urbanização, com centros urbanos vinculados ao dinamismo da atividade rural e por ele impulsionados (MOURA \& MAGALHÃES, 1996). Nos anos 1980, devido à industrialização e à mecanização agrícola, houve significativa perda da população rural e o aumento do número de núcleos urbanos. Nessa década a população urbana ultrapassou a população rural no Estado do Paraná. (OLIVEIRA, 2001).

No entanto, conforme PIFFER (1999), as mudanças ocorridas no Estado do Paraná e a compreensão do crescimento da região Oeste relacionam-se diretamente com a dinâmica da população. Essa dinâmica influiu na formação da estrutura produtiva regional. Nesse sentido, para compreender uma região é preciso compreender a localização da população e a forma como ela influi na ocupação do espaço regional.

\footnotetext{
* Doutor em Demografia pela Universidade Estadual de Campinas (UNICAMP). Professor adjunto do Colegiado de Economia da Universidade Estadual do Oeste do Paraná (UNIOESTE)/Campus de Toledo. Pesquisador do Grupo de Estudos e Pesquisas em Agronegócio e Desenvolvimento Regional (GEPEC). Endereço Eletrônico: rippel@unioeste.br

** Ph.D. em Desenvolvimento Regional pela Université du Québec à Chicoutimi (UQAC)- Canadá. Professor adjunto do Colegiado de Economia na Universidade Estadual do Oeste do Paraná (UNIOESTE)/Campus de Toledo. Pesquisador do Grupo de Estudos e Pesquisas em Agronegócio e Desenvolvimento Regional (GEPEC). Pesquisador associado do GRIR-UQAC. Endereço eletrônico: jandir@unioeste.br
} 
Neste sentido e, concordando com as proposições de Cunha (2003), sustenta-se que a análise do Oeste do Paraná fornece um cenário interpretativo bastante interessante do ponto de vista de compreensão das várias formas pelas qual a migração se entrelaça com fatores econômicos e acelera ou retarda um processo de desenvolvimento regional.

De modo que, objetivo desse artigo é analisar alguns dos principais fatores que influenciaram o crescimento e a localização da população no Oeste paranaense, particularmente a população rural. Essa averiguação utiliza como objeto de estudos a população da área, dadas algumas particularidades ali presentes, dentre elas: a dispersão relativa da população rural no espaço regional, conforme apontado nos estudos de Rippel (2005); o esgotamento tardio da fronteira agrícola nessa área no escopo da região Sul do Brasil; a dispersão espacial dos núcleos urbanos atrelados a um continum urbano-rural no entorno de núcleos urbano-industriais, o que denota uma particularidade no contexto paranaense. Nesse sentido, essa análise fornecerá um sistema de informações útil para explicar as tendências do crescimento da população urbana e rural nos últimos decênios do século XX. Informações estas que serão elementos para análises comparativas da dinâmica populacional nas regiões cujo esgotamento da fronteira agrícola ocorreram nos últimos vinte anos.

\section{AS MEDIDAS DE LOCALIZAÇÃO DA POPULAÇÃO URBANA E RURAL}

Os primeiros pesquisadores a aplicar e sistematizar o método diferencial-estrutural no Brasil foram: Lodder (1974) e Haddad (1989). Ambos são importantes referências atuais da aplicação empírica desse instrumental ao caso brasileiro, também nas análises voltadas ao Paraná e na Mesorregião Oeste Paranaense Ferrera de Lima et all. (2004). Outro elemento importante no método diferencial-estrutural é a delimitação da área de análise. Ao utilizar o peso relativo da população urbana e rural, o método anula o efeito "tamanho" das regiões.

Para o cálculo das medidas de localização organizaram-se as informações em uma matriz que relaciona a distribuição domiciliar-espacial de uma variável-base. No presente estudo utiliza-se a população (E) distribuída por situação de domicílio (urbana e rural) nos municípios que existiam na região nos anos 1980. Com isso, excluíram-se os municípios emancipados após esse período. As colunas mostram a distribuição da população entre os municípios, e as linhas mostram a distribuição da população por situação de domicilio de cada um dos municípios, conforme Figura 1. O dado básico para a construção do modelo é a chamada "Matriz de Informações". Tratando-se de um modelo de estática-comparada, o qual 
necessita de no mínimo, duas dessas matrizes, referindo-se uma ao período-base e outra ao ano considerado.

A Matriz de Informações (FIGURA 1) é formada em suas linhas pelos diversos setores e, nas colunas, pelas regiões.

QUADRO 1 - MATRIZ DE INFORMAÇÕES SHIFT AND SHARE

\begin{tabular}{lcccc}
\hline \multicolumn{1}{c}{ Municípios } & $\mathbf{1}$ & $\mathbf{2} \ldots$ & $\boldsymbol{j} \ldots$ & $\boldsymbol{n}$ \\
Domicílios & $E_{11}$ & $E_{12}$ & $E_{1 j}$ & $E_{1 n}$ \\
\hline 1 & $E_{21}$ & $E_{22}$ & $E_{2 j}$ & $E_{2 n}$ \\
2 & $\ldots$ & $\ldots$ & $\ldots$ & $\ldots$ \\
$\ldots$ & $E_{i 1}$ & $E_{i 2}$ & $E_{i j}$ & $E_{i n}$ \\
$\mathrm{I}$ & $\ldots$ & $\ldots$ & $\ldots$ & $\ldots$ \\
$\ldots$ & $E_{k 1}$ & $E_{k 2} \ldots$ & $E_{k j \ldots}$ & $E_{k n}$ \\
$K$
\end{tabular}

FONTE: HADDAD, 1989.

Esquematicamente,

$A=E_{i j}\left\{\begin{array}{l}i=1,2 \ldots k \\ j=1,2 \ldots n\end{array}\right\}$

Tem-se:

$A_{0}=$ ano-base e,

$A_{1}=$ ano fim do período.

$E_{i j}^{\prime \prime}=$ População no fim do período no domicilio $i$, município $j$;

$E_{i j}^{\prime}=$ População no ano-base no domicilio $i$, no município $j$;

$\propto_{i j}=$ Taxa de crescimento da população no domicilio $i$, Município $j$;

$\propto_{i t}=$ Taxa de crescimento da população no domicilio $i$ no País;

$\propto_{t t}=$ Taxa de crescimento regional da população.

Seja:

$E_{i j}^{\prime \prime}=E_{i j}^{\prime}+\Delta E_{i j}^{\prime \prime}$

Tem-se que:

$\Delta E_{i j}^{\prime \prime}=E_{i j}^{\prime \prime}-E_{i j}^{\prime}$ onde, $E_{i j}^{\prime \prime}=E_{i j}^{\prime}\left(\frac{E^{\prime \prime}}{E_{i j}^{\prime}}\right)=E_{i j}^{\prime} \cdot \alpha_{i j}$ 
Resulta:

$\Delta E_{i j}^{\prime \prime}=E_{i j}^{\prime}\left(\alpha_{i j}-1\right)$.

Considera-se agora:

$\alpha_{t t}=\frac{E^{\prime \prime}{ }_{t t}}{E^{\prime}{ }_{t t}}$ Taxa regional de crescimento da população;

$\alpha_{i t}=\frac{E^{\prime \prime}{ }_{i t}}{E^{\prime}{ }_{i t}}$ Taxa regional de crescimento da população no domicilio $i$

Pode-se somar e subtrair esses dois valores da expressão (7) que ela não se altera:

$\Delta E_{i j}^{\prime \prime}=E_{i j}^{\prime}\left(\alpha_{i j}-1+\alpha_{t t}-\alpha_{t t}+\alpha_{i t}-\alpha_{i t}\right)$

Ou trocando os termos de posição:

$\Delta E_{i j}^{\prime}=E_{i j}^{\prime}\left(\alpha_{t t}-1+\alpha_{i t}-\alpha_{t t}+\alpha_{i j}-\alpha_{i t}\right)$

Decompondo, em seguida, o segundo membro em parcelas:

$\Delta E_{i j}^{\prime \prime}=E_{i j}^{\prime}\left(\alpha_{t t}-1\right)+E_{i j}^{\prime}\left(\alpha_{i t}-\alpha_{t t}\right)+E_{i j}^{\prime}\left(\alpha_{i j}-\alpha_{i t}\right)$

Substituindo o valor de $\Delta E_{i j}^{\prime \prime}$ dado por (7) na equação (5), resulta:

$$
\begin{aligned}
& E^{\prime \prime}=E_{i j}^{\prime}+E_{i j}^{\prime}\left(\alpha_{t t}-1\right)+E_{i j}^{\prime}\left(\alpha_{i t}-\alpha_{t t}\right)+E_{i j}^{\prime}\left(\alpha_{i j}-\alpha_{i t}\right) \\
& E_{i j}^{\prime \prime}-E_{i j}^{\prime}-E_{i j}^{\prime}\left(\alpha_{t t}-1\right)=E_{i j}^{\prime}\left(\alpha_{i t}-\alpha_{t t}\right)+E_{i j}^{\prime}\left(\alpha_{i j}-\alpha_{i t}\right) \\
& \left(E_{i j}^{\prime \prime}-E_{i j}^{\prime}\right)-E_{i j}^{\prime}\left(\alpha_{t t}-1\right)=E_{i j}^{\prime}\left(\alpha_{i t}-\alpha_{t t}\right)+E_{i j}^{\prime}\left(\alpha_{i j}-\alpha_{i t}\right)
\end{aligned}
$$

A equação (9) fornece os valores correspondentes a cada efeito definido pelo modelo. Explicam-se cada um desses efeitos e sua fórmula:

a) $V L T_{i j}=\left(E_{i j}^{\prime \prime}-E_{i j}^{\prime}\right)-E_{i j}^{\prime}\left(\alpha_{t t}-1\right)$

Ou seja, é a diferença entre a variação efetiva na população de $i$ em $j$ e a variação teórica da população, isto é, aquele que o domicilio $i$ teria no município, caso crescesse à taxa da região $\alpha_{t}$;

b) $V L P_{i j}=E_{i j}^{\prime}\left(\alpha_{i t}-\alpha_{t t}\right)$

A variação proporcional corresponde parte da VLT causada por uma re-alocação de atividades (para domicilio $i$ ou do Município j). Pela própria fórmula, observa-se que a diferença entre as taxas domiciliar e regional indica que o Município possui vantagens comparativas para o desenvolvimento do domicilio;

c) $V L D_{i j}=E_{i j}^{\prime}\left(\alpha_{i j}-\alpha_{i t}\right)$ 
A $V L D$ corresponde, ao contrário, aquela parte do efeito total determinada por uma maior ou menor participação no crescimento domiciliar a nível regional. Um sinal positivo para a $V L D$ se explica pela especialização regional em domicílios dinâmicos.

Simbolicamente, tem-se para o domicilio $i$, na Município $j$ :

$$
V L T_{i j}=V L D_{i j}+V L P_{i j}
$$

Interessa conhecer esses efeitos em nível regional. Para tanto, basta somar os valores encontrados para os $k$ setores:

$$
\frac{k}{\sum_{i=1} V L T_{i j}}=\frac{k}{\sum_{i=1} V L D_{i j}}+\frac{k}{\sum_{i=1} V L P_{i j}}
$$

Tem-se então:

$$
V L T_{j}=V L D_{j}+V L P_{j}
$$

A equação (10) é idêntica à equação (9), apenas os símbolos foram modificados.

$\mathrm{Na}$ aplicação ao caso demográfico regional, utilizaram-se ambas as equações; a (9) para fins de cálculo dos valores, e a (10) para a interpretação dos dados.

\section{O PERFIL DO CRESCIMENTO POPULACIONAL REGIONAL}

De uma sociedade exclusivamente agrária de meados do século XX, o Oeste paranaense chegou ao inicio do século XXI como uma região urbanizada de expressiva densidade populacional. $\mathrm{Na}$ área, a partir de 1970, iniciou-se uma forte expansão da rede urbana, que passou a funcionar estritamente vinculada ao dinamismo da atividade rural e por ela impulsionada. Da mesma forma, nos anos 1980, devido à industrialização e à mecanização agrícola, houve significativa perda da população nas áreas rurais e o crescimento acelerado das cidades. Entre 1970 e 2000 o contingente populacional da região Oeste paranaense aumentou em 51,18\%. No entanto, o fato mais marcante foi que a população urbana apresentou uma evolução de 520,92\% no mesmo período. Ao contrário, a população rural demonstrou uma redução de $-65,31 \%$ no período analisado. Os principais fatores responsáveis pela diminuição da população rural foram a significativa reestruturação da base produtiva da região, ocasionada pela modernização da base técnica de produção, e o esgotamento da fronteira agrícola. Por isso, as mudanças populacionais na região Oeste do Paraná relacionam-se 
diretamente com a dinâmica do espaço rural. Essa dinâmica influi na formação da estrutura produtiva regional (FERRERA DE LIMA et all, 2003).

Atualmente, a região está economicamente embasada nas atividades da pecuária e da agricultura moderna de exportação, que impulsionam o comércio e a economia regional, fornecendo as bases para implantação de indústrias, principalmente as ligadas ao setor agropecuário. Isto se traduz em efeitos de difusão da base de exportação sobre a economia da área, provocando um expressivo movimento de capitais, de serviços e de pessoas, principalmente nas áreas urbanas da região, notadamente nos maiores municípios. Esse movimento refletiu no acelerado crescimento das cidades da região, e possui uma clara dependência das atividades agroindustriais da área, que por sua vez, induzem o crescimento de atividades econômicas complementares.

Nesse panorama os fatores demográficos endógenos exercem papel preponderante no processo, e têm características muito particulares na região, inerentes às peculiaridades da sua ocupação e de desenvolvimento. Tais particularidades da região estão fortemente ligadas à sua própria formação econômica, política e social, oriundas da forma de ocupação recente e da produção em pequenas e médias propriedades rurais, assim como passaram a ter coesão no discurso político dos empresários e das autoridades regionais para reivindicações em prol da região (Rippel, 2005).

Entretanto, rapidamente o Oeste do PR se converteu numa área em que substantivas transformações na sua economia e no seu sistema produtivo deflagraram intensos processos de esvaziamento das áreas rurais, exacerbação dos movimentos de urbanização e expressivas perdas populacionais da área com um todo via emigração. É evidente que esses processos, nos seus diversos âmbitos de operação, interestadual, intra-estadual, e intra-regional desenvolveram-se, muitas vezes, de modo heterogêneo e com diferentes intensidades, imprimindo dinâmicas diferenciadas de crescimento populacional, e provocando reordenamentos contínuos da distribuição espacial da população da área. 
TABELA 1 - VARIAÇÃO DIFERENCIAL - ESTRUTURAL DO CRESCIMENTO DA POPULAÇÃO URBANA E RURAL DE ALGUNS MUNICÍPIOS DO OESTE DO PARANÁ - 1980 E 2000.

\begin{tabular}{|c|c|c|c|c|}
\hline \multirow{2}{*}{$\begin{array}{l}\text { DOMICÍLIO } \\
\text { /MUNICÍPIO }\end{array}$} & \multicolumn{2}{|c|}{ VARIAÇÃO ESTRUTURAL } & \multicolumn{2}{|c|}{ VARIAÇÃO DIFERENCIAL } \\
\hline & URBANA & RURAL & URBANA & RURAL \\
\hline Foz do Iguaçu & 91.356 & -92.944 & 102.868 & -46.748 \\
\hline Cascavel & 89.741 & -91.301 & 39.256 & 46.266 \\
\hline Toledo & 53.627 & -54.559 & 15.987 & -4.922 \\
\hline Mal. C. Rondon & 41.150 & -41.865 & -37.656 & -7.061 \\
\hline Assis Chat. & 32.598 & -33.164 & -34.807 & -6.093 \\
\hline Medianeira & 26.918 & -27.386 & -15.375 & -4.656 \\
\hline Formosa Oeste & 26.355 & -26.813 & -51.032 & -10.635 \\
\hline São M. Iguaçu & 25.071 & -25.507 & -3.685 & -1.974 \\
\hline Cap. L. Marques & 21.977 & -22.359 & -16.191 & -7.132 \\
\hline Guairá & 21.354 & -21.725 & -18.824 & -1.303 \\
\hline Corbélia & 21.023 & -21.388 & -24.940 & -7.029 \\
\hline Guaraniaçu & 20.721 & -21.081 & -19.421 & -671 \\
\hline Palotina & 20.679 & -21.039 & -8.522 & -3.182 \\
\hline Matelândia & 18.664 & -18.989 & -18.663 & -4.881 \\
\hline Santa Helena & 18.482 & -18.803 & -2.641 & 2.502 \\
\hline Terra Roxa & 18.459 & -18.780 & -22.308 & -1.942 \\
\hline Santa Tereza Oeste & 17.936 & -18.247 & -36.814 & 1.705 \\
\hline Nova Aurora & 13.462 & -13.696 & -8.933 & -1.093 \\
\hline Três Barras PR & 13.092 & -13.320 & -10.501 & 841 \\
\hline Catanduvas & 10.785 & -10.973 & -8.016 & 757 \\
\hline Vera Cruz Oeste & 10.208 & -10.385 & -11.270 & -1.235 \\
\hline Missal & 9.217 & -9.377 & 4.551 & 1.078 \\
\hline Sta. Terezinha Itaipu & 8.442 & -8.589 & 2.022 & 1.316 \\
\hline Céu Azul & 8.418 & -8.565 & -6.900 & 1.138 \\
\hline Boa Vista Aparecida & 7.908 & -8.046 & -5.448 & 749 \\
\hline Lindoeste & 7.525 & -7.656 & -3.212 & -33 \\
\hline Tupãssi & 7.396 & -7.524 & -7.366 & 300 \\
\hline São José Palmeiras & 7.055 & -7.178 & -6.397 & -1.961 \\
\hline Ouro Verde Oeste & 5.877 & -5.980 & -5.476 & -356 \\
\hline Diamante Oeste & 5.806 & -5.907 & -1.544 & -557 \\
\hline Braganey & 5.259 & -5.350 & -3.927 & 1.599 \\
\hline Nova Santa Rosa & 5.051 & -5.139 & 2.057 & 1.299 \\
\hline Campo Bonito & 4.511 & -4.590 & 2.490 & 694 \\
\hline Cafelândia & 4.476 & -4.554 & 15.290 & 1.105 \\
\hline Ibema & 2.718 & -2.766 & 5.202 & 608 \\
\hline
\end{tabular}

FONTE: IBGE (2007).

Tanto que no período de 1980 a 1990 há um movimento forte de emigração, especialmente a rural, estimulada pela conformação dos pólos econômicos de Curitiba, Londrina e Maringá, no Paraná, e de outros pólos existentes no Rio Grande do Sul, Santa Catarina e São Paulo. Esses pólos absorveram vastas quantidades de indivíduos do Oeste paranaense. Concomitantemente a este movimento, não se pode esquecer, o ocorrido com os Estados definidos como região de fronteira agrícola, que passaram também a absorver grandes levas de emigrantes do Oeste do Paraná, promovendo à transformação do perfil 
regional o qual "passou" de um perfil rural para um mais urbano no tocante a distribuição de sua população total. Já no período seguinte de 1990 a 2000, os saldos migratórios da região encolheram, mas ela ainda manteve sua tendência de perdas migratórias, mesmo como a constante capacidade dos setores urbanos da região de absorveram elevados contingentes de pessoas (RIPPEL et alli, 2005).

Pelos resultados da análise, sintetizados na tabela 1, nota-se que Foz do Iguaçu foi o município que mais tirou proveito da sua estrutura setorial e atraiu contingentes populacionais na faixa de +91.356 habitantes na área urbana, correspondentes a variação estrutural. $\mathrm{Da}$ mesma maneira, fatores próprios ao município atraíram 102.868 habitantes na área urbana. $\mathrm{O}$ inverso ocorre na área rural. Em virtude das emancipações, do impacto das atividades urbanas, da modernização agropecuária e o alagamento da Usina Hidroelétrica de Itaipu, o município de Foz do Iguaçu terá uma das maiores perdas de população rural no Oeste paranaense.

No caso de Cascavel, a evolução positiva da população rural, devida a componente diferencial, perde seu impacto em virtude dos valores negativos da componente estrutural rural. O resultado final será um saldo de -45.035 habitantes para o domićlio rural, contra +128997 dos domićlios urbanos. Além de absorver o contingente populacional rural, o município de Cascavel atraiu em torno de +83.962 habitantes para sua área urbana.

Os casos de Cascavel, Toledo, Foz do Iguaçu, Cafelândia e Ibema o movimento da população é positivo, com ganhos das áreas urbanas, no contexto de total do movimento populacional, independente dos fatores que moveram os contingentes urbanos e rurais no período. A situação foi menos desfavorável aos municípios de Marechal Candido Rondon, Nova Santa Rosa, Campo Bonito, Três Barras do Paraná, Missal e Santa Terezinha de Itaipu, cujo movimento da população ficou estável. As perdas foram compensadas com os ganhos ao longo desses vinte anos. Porém, os demais municípios tiveram perdas consideráveis de população, tanto em função das emancipações quanto do processo migratório.

Cabe chamar a atenção para o caso particular de Toledo, onde o fator diferencial foi o elemento de maior impacto no crescimento da população urbana. Enquanto a variação estrutural termina com um saldo negativo de -932 habitantes a favor da população rural, a variação diferencial, ligada a fatores estritamente locais, termina o período com um saldo positivo de +11.065 .

Apesar desses valores significativos no contexto do movimento da população, Rippel et all (2005) chamam a atenção que a localização da população rural ainda é expressiva 
no Oeste paranaense. Além disso, a região é menos urbana do que parece, em função de três elementos: O primeiro é o perfil da ocupação fundiária. A área rural do Oeste paranaense é caracterizada pela presença das pequenas propriedades até 50 ha. Essas propriedades representam 87\% do total regional segundo o Censo Agropecuário de 1996 (IBGE, 2007). O segundo elemento é a atração dos imigrantes. Nos últimos anos o Oeste paranaense recebeu diversos contingentes populacionais. Segundo RIPPEL (2005), Toledo, Cascavel e Foz do Iguaçu concentraram 48,07\% da imigração interestadual na região Oeste do Paraná no período de 1970 a 1980,60,04\% no período de 1980 a 1991, e 61,51\% no período de 1991 a 2000. No entanto, além de Toledo, Cascavel e Foz do Iguaçu, os municípios de Guaíra, Medianeira e Santa Terezinha de Itaipu estão num estágio onde a concentração da população urbana é superior à população rural. O terceiro elemento é o número de distritos existes na região. Segundo IBGE (2007), existiam no ano de 2000, 96 distritos na região Oeste do Paraná. Destes, 50 eram as sedes urbanas municipais e os 49 distritos restantes localizavam-se nos arredores dessas sedes. Cerca de $25 \%$ da população rural da região estava concentrada, no ano de 2000 , nestes distritos.

\section{CONCLUSÃO}

Como se pôde perceber o desenvolvimento da região de modo geral vinculou a dinâmica populacional e a organização do capital na área, isto porque foram estes fatores os principais responsáveis pela transformação das condições "ambientais locais", moldando-as segundo seu interesse. Como o deslocamento de pessoas e de investimentos para a área está relacionado com o comportamento da economia e com o processo de inserção e unificação dos mercados da região, a análise da relação migração, população e desenvolvimento foram evidentes no local, pois o Oeste do Paraná conjugou uma situação na qual o seu desenvolvimento deu-se conectado com a migração e o crescimento demográfico da área, isto porque a região inseriu-se no modelo de desenvolvimento nacional de ocupação de fronteiras e no processo de transnacionalização do capital agrícola, acontecimentos que impactaram diretamente no crescimento e na distribuição a população regional.

Muito embora isto tenha ocorrido apesar de que na etapa de ocupação de seu território o local atraiu uma grande leva de imigrantes, seu desenvolvimento inverteu este processo, tornando a área um lugar de elevada evasão populacional, em curto espaço de tempo. Esta evasão conforme se pode verificar deu-se fundamentalmente nas áreas rurais da 
região, sendo que o crescimento do setor urbano, apesar de expressivo não conseguiu reverter o processo.

Tanto que os dados coletados demonstram que não ocorreram mudanças significativas no padrão de localização da população urbana entre os municípios da região Oeste do Paraná. Os municípios que concentravam a população urbana no ano de 1970 continuaram concentrando durante todo o período de análise. Enquanto, a população rural está mais difusa na região. Há porém que se destacar que uma característica interessante desta região é que ainda há uma presença significativa da população rural na maioria dos municípios, com exceção de Cascavel, Foz do Iguaçu, Guaíra, Medianeira, Santa Terezinha de Itaipu e Toledo que concentraram com mais intensidade a população urbana.

Os maiores centros urbanos da região (Cascavel, Foz do Iguaçu e Toledo), se converteram num local de grandes possibilidades econômicas, não apenas em termos da valorização (e conseqüente concentração) fundiária, mas também em termos dos investimentos produtivos na agricultura para exportação e na agroindústria do complexo carne (suíno, frango e peixes). Esse movimento, atrelado à modernização do campo e à urbanização acelerada da área tornaram a região um local de forte evasão populacional favorecendo as cidades mais industrializadas, no caso Cascavel, Toledo, Medianeira, Marechal Candido Rondon e Palotina. Tanto que no contexto regional, todos os municípios têm perda de população rural. Os valores negativos da componente estrutural refletem a diferença de crescimento desse domicilio em relação ao crescimento regional. Assim, no agregado geral, o Oeste paranaense perdeu população rural, perda esta que não foi totalmente absorvida pelo crescimento das áreas urbanas.

\section{REFERÊNCIAS}

CUNHA, J. M. P. et. al. Diagnósticos regionais do Estado do Mato Grosso. Campinas: Núcleo de Estudos de População/UNICAMP, 2004. 225p. (Diagnósticos regionais do Estado do Mato Grosso, TEXTOS NEPO 49).

A redistribuição espacial da população: tendências e trajetória. Revista São Paulo em Perspectiva. Revista da Fundação SEADE/SP. v. 17., n. 3-4, jul./dez. 2003 a.

HADDAD, J. H. (Org.). Economia regional: teoria e métodos de análise. Fortaleza: BNB/ETIENE, 1989.

IBGE (Instituto Brasileiro de Geografia e Estatística). Banco de dados agregados. Disponível em: <http://www.sidra.ibge.gov.br/bda/>, Acesso em: 18 mar. 2007. 
FERRERA DE LIMA, Jandir. La diffusion spatiale du développement économique regional: l'analyse de la diffusion au sud du Brésil dans le $\mathbf{X X}^{\mathbf{o}}$ siècle. Thèse de Doctorat. DSH - Université du Québec, 2004. Disponível on-line no site Web http://www.irec.net/publications/518.pdf

FERRERA DE LIMA, J. PIACENTI, C. A.; ALVES, L. R. e PIFFER, M. A percepção do conceito de desenvolvimento regional nas áreas atingidas pelos reservatórios de Itaipu e Salto Caxias. Informe GEPEC, Unioeste Toledo (PR), v. 07, n. 1, p.49-58, 2003.

FERRERA DE LIMA, J. ; PIACENTI, C. A.; ALVES, L. R. e PIFFER, M. A localização e as mudanças da distribuição setorial do PIB nos Estados da região Sul (1970-1998). IN: CONGRESSO BRASILEIRO DE ECONOMIA E SOCIOLOGIA RURAL (SOBER), Cuiabá, Anais... Cuiabá: SOBER, 2004. 1 CD-ROM.

IBGE (Instituto Brasileiro de Geografia e Estatística). Banco de dados agregados. Disponível em: <http://www.sidra.ibge.gov.br/bda/>, Acesso em: 18 mar. 2005.

LODDER , C. A. Padrões locacionais e desenvolvimento regional. Revista Brasileira de Economia. v. 28, n. 1, Jan./Mar. 1974.

MOURA, R.; MAGALHÃES, M. V. Leitura do padrão de urbanização do Paraná nas duas últimas décadas. Revista Paranaense de Desenvolvimento. Curitiba, n. 88, set./out. 1996.

OLIVEIRA, D. Urbanização e industrialização no Paraná. Curitiba: SEED, 2001. 113 p. (Coleção historia do Paraná; textos introdutórios).

PIACENTI, C. A. et al. Análise regional dos municípios lindeiros ao lago da Usina Hidroelétrica de Itaipu. In: ENCONTRO BRASILEIRO DE ESTUDOS REGIONAIS E URBANOS, 2, 2002, São Paulo, Anais... São Paulo: ABER, 2002. 1 CD-ROM.

PIFFER, M. Apontamentos sobre a base econômica da região Oeste do Paraná. In: CASSIMIRO FILHO, F. \& SHIKIDA, P. F. A. (Orgs.) Agronegócio e Desenvolvimento regional. p. 57-84. EDUNIOESTE: Cascavel, 1999.

RIPPEL, R. Migração e desenvolvimento econômico no Oeste do estado do Paraná: uma análise de 1950 a 2000, Tese de Doutorado em Demografia, Instituto De Filosofia e Ciências Humanas da UNICAMP - Universidade Estadual De Campinas, Campinas - SP, 2005.

RIPPEL, R.; FERRERA DE LIMA; J.; ALVES, L. Notas sobre a localização da população urbana e rural no Oeste paranaense. IN: CONGRESSO BRASILEIRO DE ECONOMIA E SOCIOLOGIA RURAL (SOBER), Cuiabá, Anais... Ribeirão Preto: SOBER, 2005. 1 CDROM . 
